\title{
¿Igualdad de género en otorrinolaringología?
}

\author{
Gender equality in otolaryngology?
}

\author{
Ángela Chuang $\mathrm{C}^{1}$, Lara Ricci L1, Ximena Aguirre $\mathrm{P}^{1}$, Rodrigo Pineda $\mathrm{D}^{1}$, Andrés Nicolás N${ }^{1}$, Carla Pereira $\mathrm{P}^{1}$.
}

\begin{abstract}
RESUMEN
Introducción: Cada día son más las mujeres que ingresan a las escuelas de medicina y a los programas de especialización tradicionalmente considerados como de "predominancia masculina" (especialidades quirúrgicas y médico-quirúrgicas). Internacionalmente se han hecho esfuerzos por conocer la realidad de igualdad de género en otorrinolaringología, pero poco se sabe del escenario chileno.

objetivo: Evaluar la percepción de igualdad de género en otorrinolaringología en residentes y médicos de la especialidad en Chile. Además evaluar la asociación entre género y aspectos de vida laboral y personal.

Material y método: Elaboración y distribución de encuesta que recopila información epidemiológica, de formación, de vida personal y laboral, además de preguntas relacionadas con percepción de acoso y/o discriminación de género.

Resultados: 139 encuestados (55\% hombres, $75 \% \geq 40$ años, $72 \%$ especialistas).

Se obtuvo una diferencia estadísticamente significativa en percepción de discriminación negativa de género hacia mujeres tanto durante la residencia como en el lugar de trabajo, mayor reporte de cuestionamiento de habilidades quirúrgicas y de comentarios sexistas en desmedro de mujeres. Sin diferencia estadísticamente significativa en percepción de acoso sexual ni en variación de horas de trabajo luego de ser padre o madre.

Conclusión: En la población encuestada, la población femenina reportó mayor percepción de discriminación de género. Se deben aumentar los esfuerzos por estudiar la situación de igualdad de género en nuestro medio y generar ambientes amigables para el desarrollo profesional indiferente del género.
\end{abstract}

Palabras clave: Otorrinolaringología, sexismo, derecho de las mujeres, Chile.

\begin{abstract}
Introduction: Everyday more women enter medicine schools and to traditionally known as "male predominant" specialization programs (surgical and medical-surgical specialties). Internationally, efforts have been made to understand the reality regarding gender equality in otolaryngology, but little is known about the Chilean scene.
\end{abstract}

1 Servicio de Otorrinolaringología Hospital Barros Luco Trudeau, Santiago, Chile.

Los autores declaran no tener conflictos de interés.

Recibido el 17 de febrero, 2019. Aceptado el 4 de julio, 2019. 
Aim: To evaluate the perception on gender equality in otolaryngology of residents and physicians of the specialty in Chile. Also, to evaluate the association between gender and aspects of labor and personal life.

Material and methods: Elaboration and distribution of a survey that collects epidemiologic, schooling, personal and labor life information, additionally questions related to perception of harassment and/or gender discrimination.

Results: 139 people surveyed (55\% men, 75\% $\leq 40$ years old, $72 \%$ specialists). Statistically significant difference was found in the perception of negative discrimination against women during residency and in work places, greater report of questioning of surgical abilities and sexist comments against women. No statistical difference was found in the perception of sexual harassment nor variation of work hours after mother or fatherhood.

Conclusion: The female population surveyed reported a greater perception of gender discrimination. Efforts to study gender equality situation should be increased and friendly environments for professional development independent of gender should be generated.

Key words: Otolaryngology, sexism, women's rights, Chile.

\section{INTRODUCCIÓN}

La discriminación de género es cualquier hecho 0 acción en la que una persona reciba un trato desigual a causa de su género, pudiendo afectar tanto a hombres como mujeres. En dicho contexto se puede dar acoso laboral, entendido como agresión u hostigamiento ejercida por el empleador u otro trabajador que cause menoscabo, maltrato 0 humillación ${ }^{1}$. También puede ocurrir acoso sexual, en el que se incurre en solicitudes de carácter sexual no consentidas 0 aceptadas por la persona afectada, generando un ambiente laboral ofensivo ${ }^{1}$.

El tema de la igualdad de género en los últimos años ha ido ganando terreno en distintos ámbitos de la realidad tanto nacional como internacional. En Chile, en el año 2016 se publicó el primer informe GET: género, educación, trabajo². Este fue el primer estudio chileno sobre desigualdad de género en el ciclo vital, en él se menciona que "la tasa de participación laboral femenina es baja, hay segregación a nivel de ramas de actividad, están subrepresentadas en los altos cargos y, a pesar que existe una legislación que la prohíbe, enfrentan una brecha salarial de género que es de las más altas del mundo"2. Este mismo informe también trata la temática de la maternidad y su impacto en la carrera profesional, exponiendo que social y legislativamente se pone el peso y el costo de la parentalidad sobre la mujer².
Asimismo, en su versión del año 2018 (Informe GET 2018: avances, contrastes y retos de tres generaciones) ${ }^{3}$ destaca que "se observa el surgimiento de una nueva ola feminista ...lográndose abrir un debate público que convoca a la sociedad entera, obligando a mirar de frente patrones de conducta... como lo son el acoso y abusos sexuales, pero también las discriminaciones a las que se somete diariamente a las mujeres..."3.

En el ámbito de la medicina, la primera Escuela de Medicina fue fundada en nuestro país en 1842 por la Universidad de Chile y 45 años más tarde, en 1887, se titula la primera médica de Chile (y de América del Sur $)^{4}$. Desde entonces, han sido grandes pasos los que ha dado el género femenino en la medicina en términos de igualdad. Cada día son más las mujeres que ingresan a las escuelas de medicina y a los programas de especialización tradicionalmente considerados como de "predominancia masculina" (especialidades quirúrgicas y médico-quirúrgicas) ${ }^{5}$. Sin embargo, persisten aún dudas respecto a la igualdad en el trato y en oportunidades en este escenario.

Existen publicaciones que abordan el tema de género en esta especialidad desde 1980, donde se ha evidenciado, un aumento progresivo de la representación femenina en la especialidad y en la autoría en revistas científicas ${ }^{6}$. Sin embargo, cabe destacar que el incremento anual de mujeres que ingresan a programas de especialización en 
otorrinolaringología es significativamente menor que el incremento observado en estudiantes universitarios, estudiantes de medicina y residentes en general en Estados Unidos entre los años 1975 y 20107. Un estudio en el año 2010, que encuestó a mujeres miembros de la Academia Americana de Otorrinolaringología: Cirugía de Cabeza y Cuello (AA0-HNS, por su sigla en inglés), reveló que 33\% de las mujeres reportó un cambio de su práctica clínica en el pasado, y la mitad de estos cambios se debió a acoso o discriminación8.

En este escenario, en el año 2010 la AAO-HNS creó la sección Women in Otolaryngology (mujeres en otorrinolaringología) que tiene como objetivo principal el "asistir en el desarrollo de la carrera de mujeres otorrinolaringólogas (y quienes consideran la especialidad)" (https://www.entnet.org/content/women-otolaryngology-section) $)^{9,10}$. Respecto a la realidad nacional, en un estudio de Cardemil y cols. se reportó que al año 2011, solo el 20,1\% de los otorrinolaringólogos contratados en el servicio público y $24 \%$ de los miembros de la Sociedad Chilena de Otorrinolaringología (SOCHIORL) eran mujeres. De los datos mostrados en su estudio, observó que el único servicio de otorrinolaringología en que existía un predominio de especialistas mujeres era el Servicio Metropolitano Sur con $54 \%$ de participación femenina ${ }^{11}$.

Debido a la presión global por posicionar a la mujer en un papel de igualdad en todo ámbito y por el desconocimiento hasta ahora de la realidad de las mujeres otorrinolaringólogas en nuestro país, primero parece necesario conocer el escenario actual respecto a esta temática en nuestra especialidad, con el objetivo de impulsar eventuales cambios que permitan el desarrollo en un ambiente igualitario.

\section{OBJETIVO}

El objetivo principal de este trabajo es evaluar la percepción de igualdad de género en otorrinolaringología en residentes y médicos de la especialidad en Chile. Para esto se buscó determinar la percepción de discriminación de género, evaluar la asociación entre género y experiencia de acoso físico y/o verbal, además de conocer la experiencia respecto al cuestionamiento de las habilidades profesionales por terceros. Otros objetivos fueron: determinar ingreso económico de los especialistas y evaluar si se presentan diferencias de género y comparar la influencia de la maternidad o paternidad en la cantidad de horas destinadas al desempeño laboral.

\section{MATERIAL Y MÉTODO}

Se diseñó una encuesta con preguntas de selección múltiple, de escala Likert y con espacio para comentarios libres. Esta evaluó aspectos relacionados a información epidemiológica, formación en la especialidad, vida personal y vida laboral con énfasis en evaluación de autopercepción de acoso o discriminación de género. Dicha encuesta fue anónima y fue distribuida dentro de la red de contactos de los autores a través de una plataforma virtual (EncuestaFácilo) a otorrinolaringólogos y residentes de la especialidad. No fue posible su distribución a través de la SOCHIORL. Este estudio incluyó consentimiento informado para sus participantes y cuenta con aprobación de Comité Ético Científico del Servicio de Salud Metropolitano Sur.

\section{RESULTADOS}

De un total de 139 encuestados, 62 fueron mujeres $(45 \%)$ y 77 fueron hombres $(55 \%)$. Cuarenta y nueve por ciento de la muestra se encontró dentro del rango etario de entre 31 y 40 años, correspondiendo el $75 \%$ a menores de 40 años. De los encuestados, 100 correspondieron a especialistas otorrinolaringólogos (72\%) con $55 \%$ de hombres, mientras que $39(28 \%)$ fueron residentes de la especialidad con $56 \%$ de hombres.

\section{Percepción de discriminación de género}

Al preguntar a los encuestados respecto a la percepción de influencia de su propio género durante la residencia, la respuesta más frecuente $(60 \%)$ fue "indiferente", es decir que su género no habría tenido influencia en el trato que recibieron durante su periodo de formación. Esta fue la moda tanto en hombres (68\%) como en mujeres (50\%). Al evaluar aquellas respuestas en las que los encuestados 
Tabla 1. Descripción de la muestra

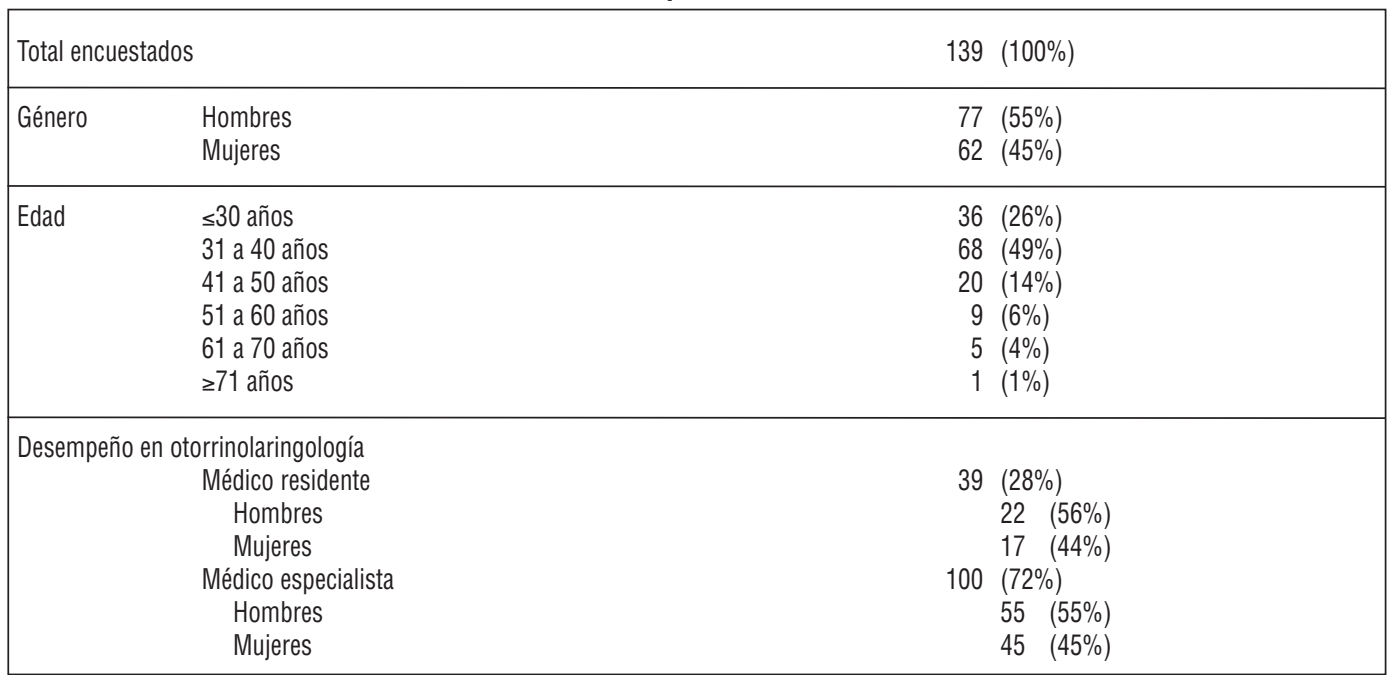

consideraron una influencia negativa de su género, se observó una mayor percepción de discriminación negativa en mujeres $(37 \%$ vs $8 \%)$, con una diferencia estadísticamente significativa $(p<0,001)$. Al evaluar la percepción de discriminación negativa en el lugar de trabajo (considerando sólo médicos especialistas) se obtuvo que $55 \%$ de las mujeres vs $37 \%$ de los hombres percibieron discriminación negativa en grado leve a moderado, mientras que $31 \%$ de las mujeres vs $0 \%$ de hombres percibieron discriminación moderada a severa. Inversamente, al evaluar a quienes respondieron sentirse "nada discriminados", fue mayor el porcentaje de hombres $(63 \%)$ quienes dieron esta respuesta vs mujeres (14\%), siendo esta diferencia también estadísticamente significativa ( $p<0,001$, Figura 1$)$.

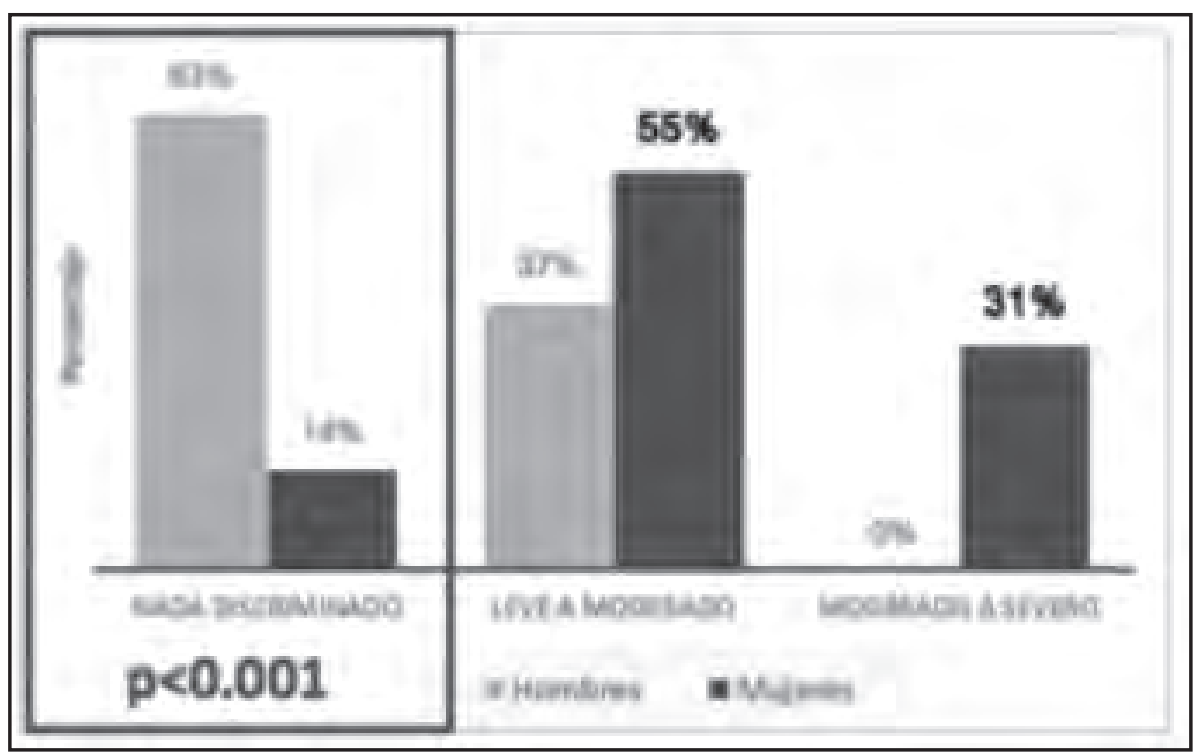

Figura 1. Percepción de discriminación de género en lugar de trabajo. 
Sobre la percepción de acoso sexual (físico y/0 verbal) en su lugar de trabajo, $17,7 \%$ de la mujeres y $15,5 \%$ de los hombres respondieron haberse sentido víctimas, sin lograr establecer una diferencia estadísticamente significativa entre ambos géneros $(p=0,73)$. Del grupo que sí había percibido acoso sexual, éste fue de predominio verbal, no obstante, sorprende el reporte de 6 encuestados víctimas de acoso físico (5 mujeres y 1 hombre; Figura 2).

Al consultar respecto a comentarios sexistas en desmedro del propio género, se vio que de forma significativa $(p<0,001)$ más mujeres reportaron haber sido víctimas de comentarios sexistas con $67,7 \%$, a diferencia de los hombres que solo manifestaron este tipo de comentarios en 16,9\%.
Las mujeres también reportaron mayor cuestionamiento de sus habilidades quirúrgicas a causa de su género (34\%), a diferencia de los hombres en que solo $3 \%$ refiere haber sido cuestionado, siendo esta diferencia estadísticamente significativa ( $p$ $<0,001$ ). De ellas, en la mayoría de los casos el cuestionamiento se originó exclusivamente por su contraparte masculina $(66,7 \%)$.

\section{Diferencia de ingreso económico por género}

Al evaluar el ingreso económico de los encuestados que aceptaron contestar este ítem, los hombres reportaron un mayor ingreso económico mensual que las mujeres ( $p<0,001$, Figura 3 ). Si bien, hombres reportaron más horas de trabajo

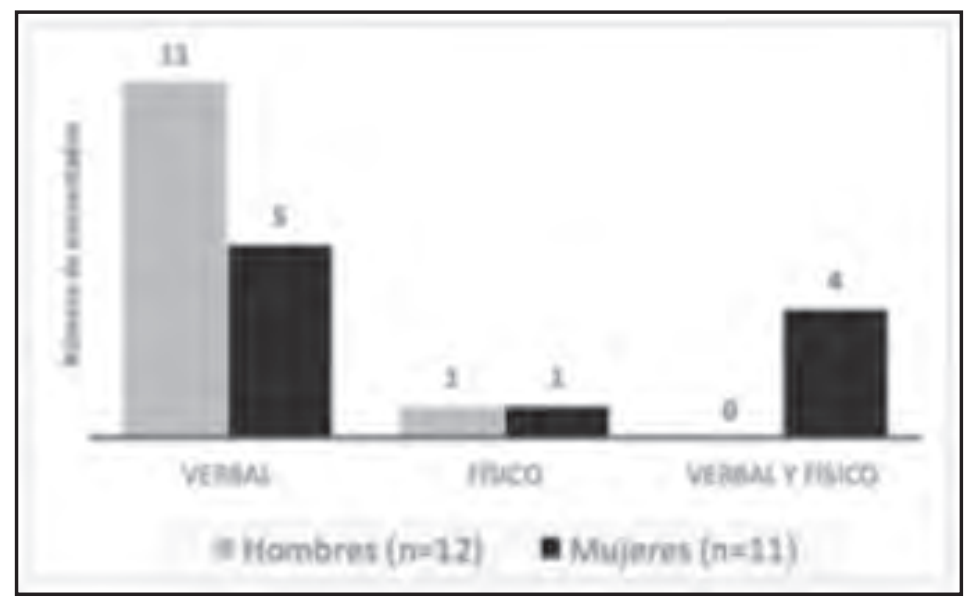

Figura 2. Percepción de acoso sexual en lugar de trabajo.

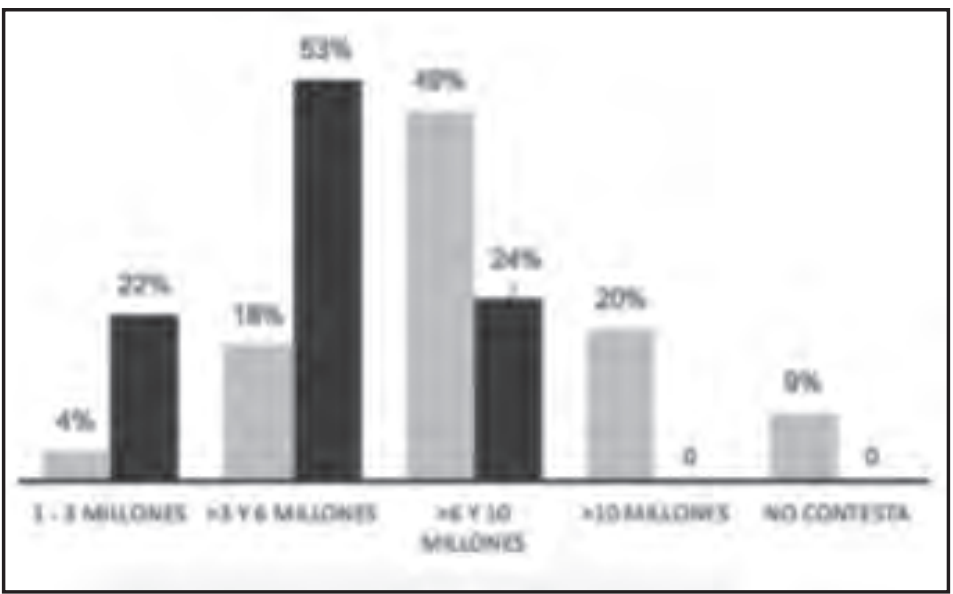

Figura 3. Ingreso económico mensual. 
semanales al comparar más de 44 horas versus menos de 44 horas $(p<0,001)$ y tanto hombres como mujeres refieren un régimen de trabajo predominante mixto (sector público y privado), por las características de la encuesta no fue posible corregir por edad, número específico de horas trabajadas ni lugar de desempeño laboral.

\section{Influencia de la maternidad o paternidad en dedicación horaria}

De los especialistas otorrinolaringólogos encuestados, 44 sujetos fueron padre 0 madre. No se observó una diferencia estadísticamente significativa entre hombres y mujeres al preguntar por la variación en la cantidad de horas de trabajo luego de ser padres $(p>0,05)$. No obstante, destaca que todas las mujeres respondieron haber disminuido 0 mantenido sus horas de trabajo semanales, mientras que sólo hombres (13\%) reportaron un aumento de sus horas laborales (Figura 4).

\section{DISCUSIÓN}

Dentro de las limitaciones de este estudio se debe mencionar la forma de distribución de la encuesta, que fue a través de correo electrónico y mensajería de texto, y sólo se logró acceder a la red de contactos de los autores y en algunos casos redistribuido por éstos, constituyendo esto un gran sesgo en la población que esta encuesta pudo alcanzar. También sólo fue posible acceder al número total de personas que contestaron el instrumento, sin tener información respecto a los que recibieron la encuesta pero no la contestaron. Asimismo, hubo una subrepresentación de la población mayor a 40 años y se debe considerar que los resultados presentados son predominantemente subjetivos y realizados en un momento de gran contingencia del tema de la discriminación de género, pudiendo esto también generar sesgo.

Teniendo en cuenta las limitaciones declaradas, de acuerdo a los resultados obtenidos en este estudio, en líneas generales las mujeres reportaron mayor percepción de discriminación negativa de género tanto durante su período de formación como en sus lugares de trabajo. Esto se vio reflejado también en el mayor reporte de comentarios sexistas en su desmedro y en mayor porcentaje de reporte de cuestionamiento de sus habilidades quirúrgicas. Llama la atención que $34 \%$ de las encuestadas estimó que sus habilidades quirúrgicas fueron cuestionadas a causa de su género, siendo

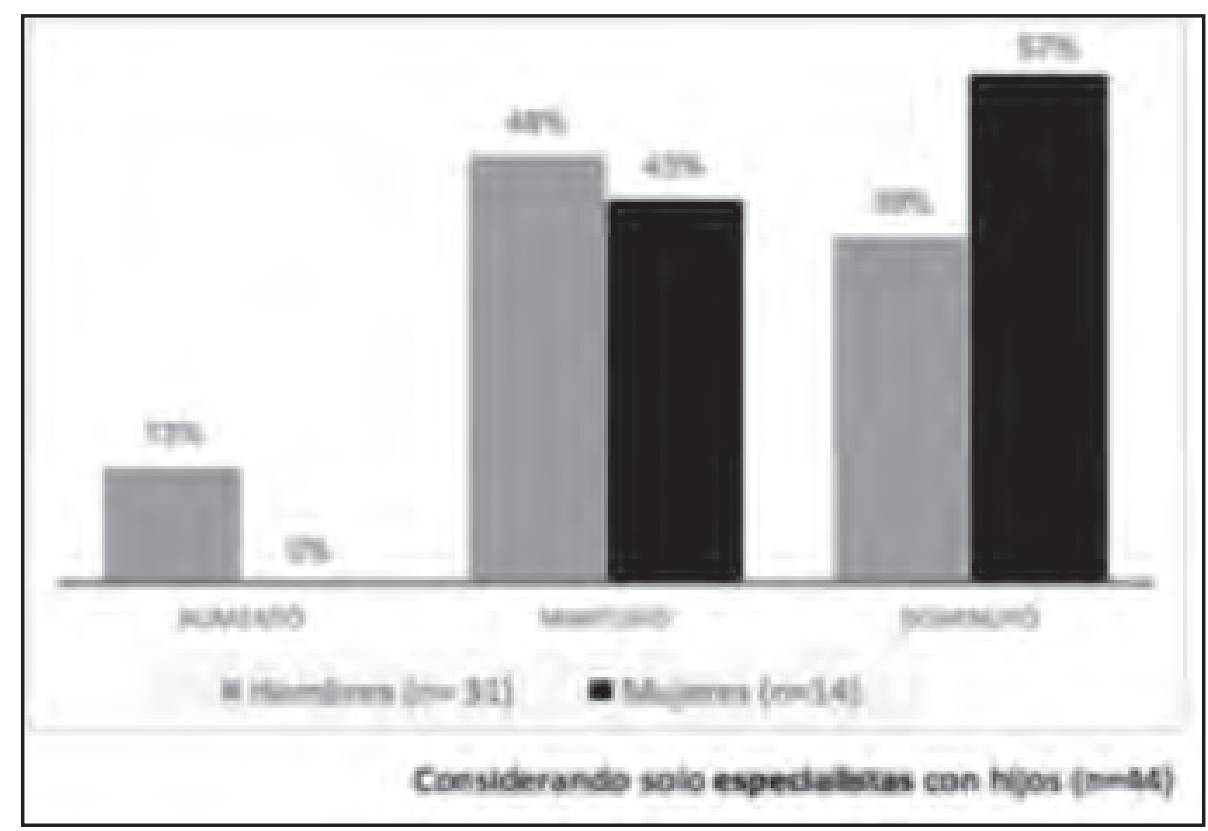

Figura 4. Variación de horas de trabajo luego de paternidad o maternidad. 
que éste en teoría es irrelevante en la apreciación profesional.

En cuanto al ingreso económico, en nuestro estudio sólo se pudo determinar de manera grosera que hombres tendrían un mayor ingreso monetario que mujeres, sin ser posible ajustar -por las características de la encuesta- por otras variables, siendo ésta una gran limitación de diseño. En el estudio de Grandis y cols (2004) ${ }^{12}$ en el que se envió una encuesta a miembros de la AAO-HNS, se obtuvo que mujeres remuneran $15 \%$ a $20 \%$ menos que hombres al ajustar por horas profesionales, horas de cirugía, tipo de práctica y años de experiencia. Asimismo, reportaron que hombres tendrían mayores ingresos, pero trabajando más de 60 horas semanales y con más horas quirúrgicas que mujeres.

En la literatura internacional publicada, en el mismo estudio citado de Grandis (2004) ${ }^{12}$ determinaron que las otorrinolaringólogas tienen más probabilidades de divorcio, de tener menos hijos y de reducir sus horas de trabajo a mayor cantidad de hijos. Concluyen además que las mujeres de la especialidad tendrían más responsabilidades familiares, afectando su avance profesional. Contrariamente, otorrinolaringólogos se apoyarían más en su pareja en las responsabilidades domésticas y cuidados de hijo(s), teniendo además más probabilidad de tener formación en alguna subespecialidad. A pesar de lo anterior, otros estudios como el

\section{BIBLIOGRAFÍA}

1. Código del Trabajo. Reconócese la función social que cumple el trabajo y la libertad de las personas para contratar y dedicar su esfuerzo a la labor lícita que elijan. Ministerio del Trabajo. Artículo 2, Inciso 2, Dictamen 3519/034. 2012. Diario Oficial de la República de Chile

2. Comunidad Mujer. Género, Educación y Trabajo: la brecha persistente. Primer estudio sobre la desigualdad de género en el ciclo de vida. Una revisión de los últimos 25 años. Informe GET. 2016.

3. Comunidad Mujer. Género, Educación y Trabajo. Avances, contrastes y retos de tres generaciones. Informe GET. 2018. de Choi y cols (2012) ${ }^{13}$ reportan que las otorrinolaringólogas representarían el $11 \%$ de los miembros de la AAO-HNS y que habría una representación de género proporcional en puestos de liderazgo, esto considerando género y edad. También Bergeron y cols $(2012)^{14}$ concluyeron que la autoría femenina en revistas de alto impacto de otorrinolaringología ha aumentado de manera constante y significativa. Entre los años 1998 a 2008 subió de 12,9\% a $21,3 \%$. También otros autores como Eloy y cols ${ }^{15}$, López y $\operatorname{cols}^{16}$ y Tomei y cols ${ }^{17}$ determinaron que la productividad femenina en especialidades quirúrgicas inicialmente es menor, pero que avanzada en su trayectoria esta iguala 0 incluso supera a la masculina.

\section{CONCLUSIÓN}

La literatura internacional sugiere la existencia de desigualdad de género en otorrinolaringología. En este estudio, como aproximación inicial a la realidad chilena, establece la existencia de -al menos- una percepción de discriminación negativa por parte de las mujeres. Se deben aumentar los esfuerzos por estudiar la situación de igualdad de género en nuestro medio, abrir la discusión respecto a la problemática de género y generar ambientes académicos amigables para desarrollar líderes en la especialidad, indiferente de su género ${ }^{18}$.

4. Facultad de Medicina Universidad de Chile. Reseña histórica. Disponible en: http://www. medicina.uchile.cl/facultad/historia/resenahistorica/114076/aumentar. Fecha de consulta: 17 febrero 2019.

5. Burgos CM, Josephson A. Gender differences in the learning and teaching of surgery: a literature review. Int J Med Educ 2014; 5:110-24.

6. Konstantinidou S, Adams M. Women in otorhinolaryngology: a historical perspective. $J$ Laryngol Otol 2018; 132: 670-2.

7. Schwartz JS, Young M, Velly AM, Nguyen LhP. The evolution of racial, ethnic, and gender diversity in US otolaryngology residency programs. Otolaryngol Head Neck Surg 2013; 149: $71-6$. 
8. BRodsky L. Women in otolaryngology are on the move: AAO- HNS recognizes section status. ENT Today. 2011. Disponible en: http://www. entto day.org/article/women-in-otolaryngologyare-on-the-move-aao- hns-recognizes-sectionstatus. Fecha de consulta: 30 Junio 2019.

9. O'Connell Ferster AP, Hu A. Women in Otolaryngology. Otolaryngol Head Neck Surg 2017; 157: 173-4.

10. American Academy of Otolaryngology. Women in Otolaryngology. Disponible en: https://www. entnet.org/content/women-otolaryngologysection. Fecha de consulta: 17 de febrero 2019.

11. Cardemil F, Barría T, Rahal M, Rodríguez R. Cantidad y distribución de otorrinolaringólogos en los sectores público y privado de salud en Chile. Rev Otorrinolaringol Cir Cabeza Cuello 2013; 73: 25-32.

12. Grandis JR, Gooding WE, Zamboni BA, Wagener MM, Drenning SD, Miller L, Doyle KJ, Mackinnon SE, Wagner RL. The gender gap in a surgical subspecialty. Arch Otolaryngol Head Neck Surg 2004; 130: 695-702.
13. Chol SS, MilleR RH. Women otolaryngologist representation in specialty society membership and leadership positions. Laryngoscope 2012; 122: 2428-33.

14. Bergeron JL, Wilken R, Miller ME, Shapiro NL, BHATTACHARYYA N. Measurable progress in female authorship in otolaryngology. Otolaryngol Head Neck Surg 2012; 147: 40-3.

15. Eloy JA, Svider P, Chandrasekhar SS, et al. Gender disparities in scholarly productivity within academic otolaryngology departments. Otolaryngol Head Neck Surg 2013; 148: 215-22.

16. López SA, Svider PF, Misra P, et al. Gender differences in promotion and scholarly impact: an analysis of 1460 academic ophthalmologists. J Surg Educ 2014; 71: 851-9.

17. Tomel KL, Nahass MM, Husain Q, et al. A genderbased comparison of academic rank and scholarly productivity in academic neurological surgery. J Clin Neurosci 2014; 21: 1102-5.

18. Megumi Hirayama M and Fernando S. Organisational barriers to and facilitators for female surgeons' career progression: a systematic review. J $R$ Soc Med 2018; 111: 324-34.

Correspondencia: Ángela Chuang Chuang Av. José Miguel Carrera 3204, San Miguel 